\title{
Echoes in Space and Time
}

\author{
Kang Lin, ${ }^{1}$ Peifen Lu, ${ }^{1}$ Junyang Ma, ${ }^{1}$ Xiaochun Gong, ${ }^{1}$ Qiying Song, ${ }^{1}$ Qinying Ji, ${ }^{1}$ Wenbin Zhang, \\ Heping Zeng, ${ }^{1, *}$ Jian Wu, ${ }^{1,2, \dagger}$ Gabriel Karras, ${ }^{3}$ Guillaume Siour, ${ }^{4}$ Jean-Michel Hartmann, ${ }^{4}$ \\ Olivier Faucher, ${ }^{3, \$}$ Erez Gershnabel, ${ }^{5}$ Yehiam Prior, ${ }^{5,8}$ and Ilya Sh. Averbukh ${ }^{5, \|}$ \\ ${ }^{1}$ State Key Laboratory of Precision Spectroscopy, East China Normal University, Shanghai 200062, China \\ ${ }^{2}$ Collaborative Innovation Center of Extreme Optics, Shanxi University, Taiyuan, Shanxi 030006, China \\ ${ }^{3}$ Laboratoire Interdisciplinaire CARNOT de Bourgogne, UMR 6303 \\ CNRS-Université Bourgogne Franche-Comté, BP 47870, 21078 Dijon, France \\ ${ }^{4}$ Laboratoire Interuniversitaire des Systèmes Atmosphériques (LISA) CNRS (UMR 7583), \\ Université Paris Est Créteil, Université Paris Diderot, Institut Pierre-Simon Laplace, \\ Université Paris Est Créteil, 94010 Créteil Cedex, France \\ ${ }^{5}$ AMOS and Department of Chemical Physics, Weizmann Institute of Science, Rehovot 76100, Israel
} (Received 31 July 2016; published 16 December 2016)

\begin{abstract}
Echo in mountains is a well-known phenomenon, where an acoustic pulse is mirrored by the rocks, often with reverberating recurrences. For spin echoes in magnetic resonance and photon echoes in atomic and molecular systems, the role of the mirror is played by a second, time-delayed pulse that is able to reverse the flow of time and recreate the original impulsive event. Recently, alignment and orientation echoes were discussed in terms of rotational-phase-space filamentation, and they were optically observed in laserexcited molecular gases. Here, we observe hitherto unreported fractional echoes of high order, spatially rotated echoes, and the counterintuitive imaginary echoes at negative times. Coincidence Coulomb explosion imaging is used for a direct spatiotemporal analysis of various molecular alignment echoes, and the implications to echo phenomena in other fields of physics are discussed.
\end{abstract}

DOI: 10.1103/PhysRevX.6.041056

\section{INTRODUCTION}

In 1950, Hahn reported [1] that if a spin system is irradiated by two properly timed and shaped pulses, a third pulse appears at twice the delay between the first two. The intuitive explanation was given in terms of time reversal; namely, the second pulse reverses the direction of propagation of the original excitation, leading to the reappearance of the original impulse [2]. In the absence of interaction with the environment, the full original excitation is recovered, but with environmental influences, various dephasing and energy-loss processes may be probed. Following the original discovery in the realms of spins, echoes were observed in many other nonlinear physical situations such as photon echo [3], cyclotron echo [4], plasma-wave echo [5], echoes in cold atoms [6,7], cavity QED [8], and even in particle accelerators $[9,10]$. Echoes form the basis for many modern

\footnotetext{
*hpzeng@phy.ecnu.edu.cn

†wu@phy.ecnu.edu.cn

olivier.faucher@u-bourgogne.fr

§yehiam.prior@weizmann.ac.il

"ilya.averbukh@weizmann.ac.il
}

Published by the American Physical Society under the terms of the Creative Commons Attribution 3.0 License. Further distribution of this work must maintain attribution to the author(s) and the published article's title, journal citation, and DOI.
Subject Areas: Atomic and Molecular Physics, Chemical Physics, Optics methodologies ranging from magnetic resonance imaging (MRI) [11] to short-wavelength radiation generation in freeelectron lasers [12-15]. Echoes are classical phenomena that are different from another well-known effect: quantum revivals [16-18], which are caused by the energy quantization of physical systems. Recently, a new type of echo was introduced: the molecular alignment echo $[19,20]$. When a gas of molecules undergoes excitation by an ultrashort laser pulse, the molecules experience a torque, causing transient alignment of the ensemble along the laser polarization axis (for a review of laser molecular alignment, see Refs. [2124]). A pair of time-delayed laser pulses results in three alignment events: two of them immediately following each excitation, and a third one, an echo, that appears with a delay equal to that between the exciting pulses. This delay can be shorter than the rotational revival time, so the echo provides access to rapidly dephasing molecular dynamics. The formation of these echoes is caused by the kick-induced filamentation of the rotational phase space [19], a phenomenon well known in the physics of particle accelerators [25]. Moreover, fractional echoes were predicted and observed in molecular alignment [20], at times that are rational fractions of the delay between the pulses. While the primary (full) alignment echo was observed by measuring the laserinduced birefringence [19], for the fractional echoes, higher-order moments of the molecular angular distribution are needed. Optical detection of the lowest-order $(1 / 2)$ 
fractional echo demanded measurement of the third harmonic of a probe pulse interrogating the kicked molecules [20], and even higher harmonics are required for the more complex higher-order fractional echoes. This requirement limits the feasibility of using purely optical techniques to probe the details of the rotational echo process.

In the present work, we overcome this obstacle by providing direct access to the spatiotemporal molecular dynamics with femtosecond time resolution, by means of the cold target recoil ion momentum spectroscopy (COLTRIMS) technique [26]. This method provides an invaluable addition to the toolbox for diagnostics of molecular evolution. Recently, this approach was successfully applied to visualizing molecular unidirectional rotation [27], with similar results reported by the Ohshima group [28]. Our current experimental arrangement is shown in Fig. 1, with details given in the Sec. 1 of the Appendix and in Ref. [27]. Here, as described above, the molecular excitation is achieved using two laser pulses. At times when the system is to be probed, a strong circularly polarized pulse is used to Coulomb-explode the molecules. The detected flying fragments provide a signature of the spatial orientation of the molecule at the time it was exploded. Our COLTRIMS setup enables us to spatiotemporally observe full and fractional echoes of high order for the first time. Building upon the angular resolution provided by COLTRIMS, we predict and observe "rotated echoes," which are alignment echoes whose angular orientation is controlled by the polarization directions of the first two pulses. We further predict "imaginary alignment echoes," which should appear at negative time delays. This counterintuitive phenomenon is explained by analytically continuing the rotational dynamics induced by the two pulses to negative evolution times. Clearly, such a "time machine" regime is not possible in classical physics, but the phenomenon of quantum rotational revivals provides a unique opportunity to study echoes

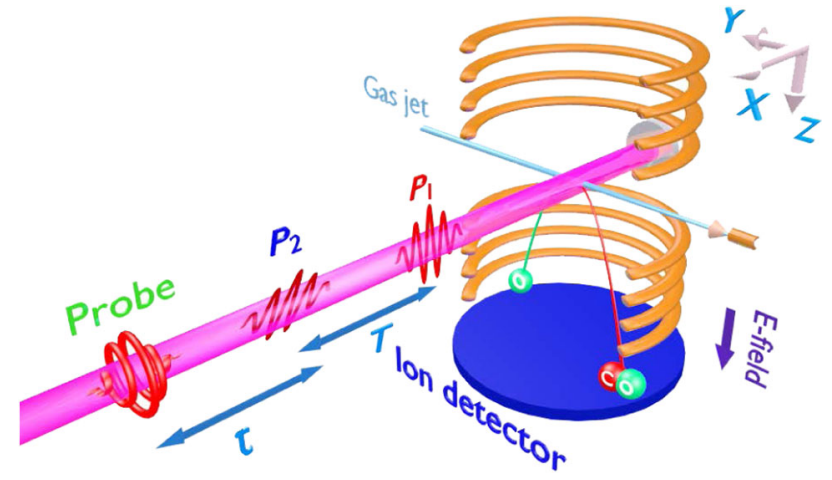

FIG. 1. Experimental setup. A supersonic gas jet of $\mathrm{CO}_{2}$ molecules subject to a pair of polarization-skewed and timedelayed femtosecond laser pulses in an ultrahigh vacuum chamber of COLTRIMS is used to create molecular alignment echoes. By scanning the delay of an intense circularly polarized probe pulse, snapshots of the angular distribution of the molecular axis at various times are taken via Coulomb explosion. at negative time; here, we report the first experimental observation of the imaginary echoes.

In what follows, we start with a discussion of the spatiotemporal dynamics of the various echoes. We then report the COLTRIMS-enabled experimental observation of the full, fractional $(1 / 2$ and $1 / 3)$, rotated, and imaginary echoes. All observations are compared with classical and quantum-mechanical simulations, within the twodimensional (2D) and three-dimensional (3D) frameworks.

\section{DYNAMICS OF ECHO FORMATION}

For the clarity of presentation, we start here with a simple 2D model. Consider an ensemble of symmetric linear molecular rotors uniformly dispersed in angle $\theta$ and with a Gaussian distribution spread $(\sigma)$ in angular velocity $\omega[19,20]$. At time $t=0$, a short, nonresonant linearly polarized laser pulse (delta kick) is applied to the gas. The interaction potential is $V(\theta, t)=-(\Delta \alpha / 4) E^{2}(t) \cos ^{2} \theta$ $[29,30]$, where $\Delta \alpha$ is the polarizability anisotropy, and $E(t)$ is the envelope of the laser pulse. As is well known, such an exciting pulse leads to molecular alignment along the laser-field polarization direction $(\theta=0)$ [21-24]. The ensemble-averaged degree of alignment $\left\langle\cos ^{2} \theta\right\rangle$ is typically referred to as the alignment factor. Following the kick, the angle $\theta$ of a rotor and its angular velocity are given by

$$
\theta=\theta_{0}+\omega t, \quad \omega=\omega_{0}-\Omega \sin \left(2 \theta_{0}\right) .
$$

Here $\omega_{0}, \theta_{0}$ are the initial conditions, and $\Omega$ is proportional to the intensity of the kick. The transformation (1) is equivalent to the Chirikov-Taylor map used in studies of deterministic chaos [31]. After the pulse, the phase-space probability distribution is given by [19]

$f(\omega, \theta, t)=\frac{1}{2 \pi} \frac{1}{\sqrt{2 \pi} \sigma} \exp \left[-\frac{[\omega-\Omega \sin (2 \omega t-2 \theta)]^{2}}{2 \sigma^{2}}\right]$.

Figures 2(a) and 2(b) show the evolution of the initial distribution with time, from (a) a single centrally symmetric folded pattern appearing shortly after the kick to (b) multiple parallel filaments emerging on longer time scales, when the alignment signal $\left\langle\cos ^{2} \theta\right\rangle(t)$ takes the isotropic value $(1 / 2$ in the $2 \mathrm{D}$ case). With time, the number of these filaments grows, and since the phase-space volume is constant, their width narrows, until they become almost horizontal and uniform in density [Fig. 2(b)]. The neighboring filaments are separated in angular velocity by $\sim \pi / t$, as can be seen from Eq. (2). Such a filamentation of phase space is well known in stellar systems [32] and in accelerator physics [25], and the folding of the phasespace distribution shown in Fig. 2(a) has much in common with the bunching effect observed in particle-accelerator beams $[9,10]$. 

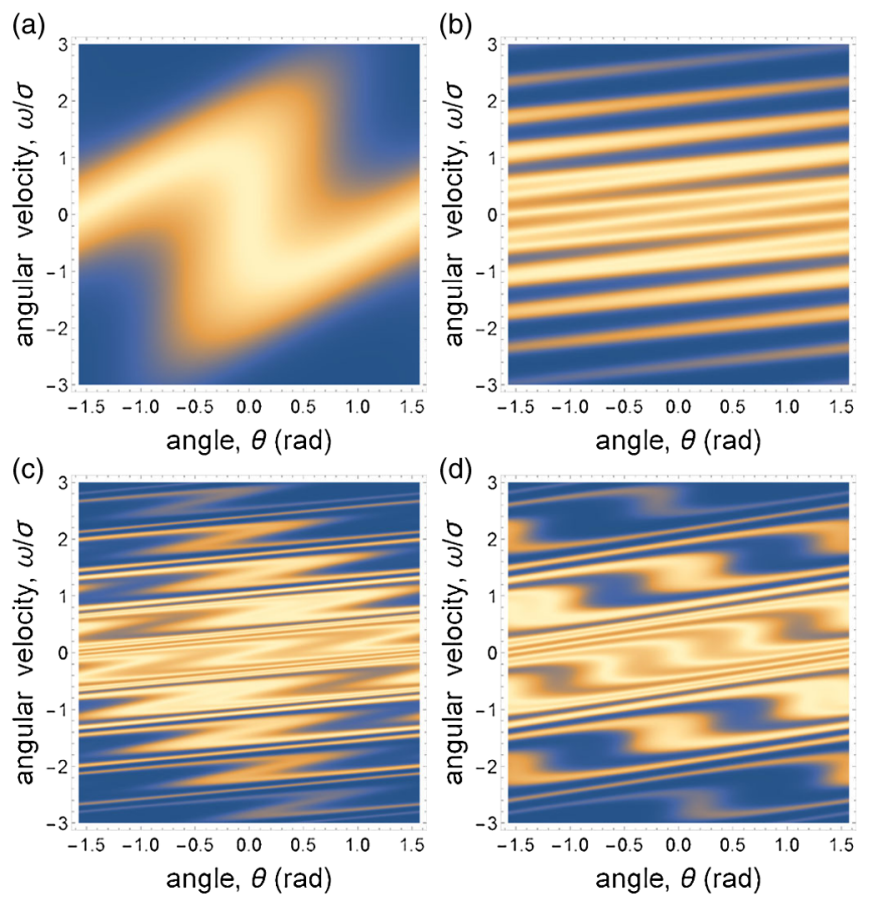

FIG. 2. Filamentation of the phase-space density distribution. For all parts of this figure, $\Omega_{1} / \sigma=1$. (a) Shortly after the kick, $\sigma t=0.5$, the density distribution folds, resulting in a transient alignment along the direction $\theta=0$. The folded pattern is centrally symmetric with respect to the phase-space point $(\theta, \omega)=(0,0)$ that is not affected by the kick. (b) On longer time scales, $\sigma t=5$, the probability density becomes wrinkled, and it develops multiple parallel filaments. (c) After a second kick is applied at $t=T$ (with $\sigma T=5$ and $\Omega_{2} / \Omega_{1}=1 / 3$ ), every filament in (b) folds in a manner similar to (a), giving rise to an alignment echo near $\theta=0$ at time $\tau \sim T$ after the second kick ( $\sigma \tau=4.56)$. (d) At $\tau \approx T / 2(\sigma \tau=2.293)$, a fractional echo is formed.

At $t=T$, the filamented ensemble is subject to a second pulse with the same linear polarization. With time, every filament in Fig. 2(b) folds in a manner similar to Fig. 2(a). Because of the "quantization" of the angular velocities, at time $\tau \sim T$ after the second kick [Fig. 2(c)], the folded parts bunch up near $\theta=0$, resulting in an alignment echo. In a similar manner, higher-order echoes are also formed at delays $2 T, 3 T, \ldots$ Moreover, as shown in Ref. [20], at rational fractions of the delay time (such as $\tau=T / 2, T / 3, \ldots)$, some highly symmetric structures in the phase space appear because of the synchronization of the folded features from non-neighboring filaments [Fig. 2(d)]. An analytical expression for the time-dependent moments $\langle\cos (2 n \theta)\rangle$, related to the expression derived in Ref. [20], completely supports these geometric arguments:

$$
\begin{aligned}
\langle\cos (2 n \theta)\rangle(\tau)= & \sum_{k=-\infty}^{k=\infty}(-1)^{k} e^{-2 \sigma^{2}(n \tau-k T)^{2}} J_{k+n}\left[2 n \Omega_{2} \tau\right] \\
& \times J_{k}\left[2 n \Omega_{1}(n \tau-k T)\right] .
\end{aligned}
$$

Here, $J_{m}(z)$ is the $m$ th-order Bessel function of the first kind. For $\sigma T \gg 1$, only positive $k$ should be taken into account. The equation describes a sequence of echoes at $\tau=(k / n) T$, where $k$ is an integer. Here, $n=1$ gives rise to the primary (full) alignment echoes, whereas for $n>1$, the equation describes the series of "fractional echoes".

The above phase-space analysis has a considerable predictive power, enabling us to anticipate several other echo phenomena-rotated and imaginary echoes (see below). As discussed in Ref. [20], for optical observation of the fractional echoes, higher moments $\langle\cos (2 n \theta)\rangle$ $(n>1)$ should be measured, which is not a trivial task. An alternative approach adopted in the present work uses Coulomb-explosion imaging of the molecular angular distribution for a direct visualization of various kinds of molecular echoes.

Rotated echoes.-Let us now consider the case where the second kick is applied (at $t=T$ ), polarized at a crossing angle $\beta$ with respect to the first one. This pulse will fold each filament in a way similar to Fig. 2(a); however, the initial position of the symmetry center of the folded features will be at $(\theta, \omega)=(\beta, m \pi / T+\beta / T)$, where $m$ is the filament number. The folded parts of the filaments will bunch up after another delay $\tau \approx T$ at the angular position

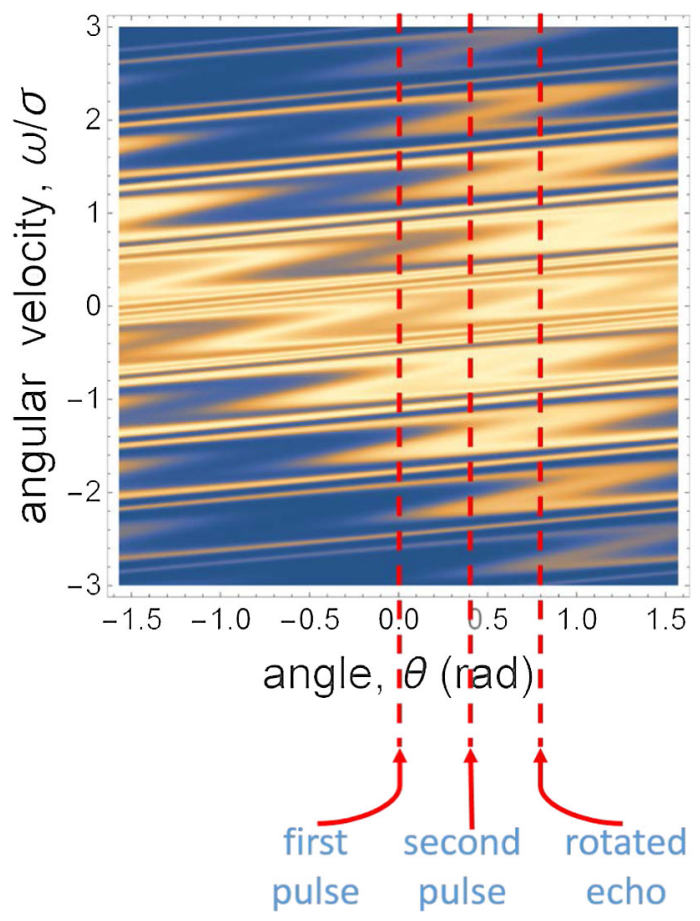

FIG. 3. Formation of the rotated echo. The polarization angles of the first and the second pulses, as well as the direction of the rotated echo are indicated by the three red dashed lines. The first pulse is applied at $t=0, \theta=0$, with $\Omega_{1} / \sigma=1$. The second pulse is applied at $t=T$ (such that $\sigma T=5$ ), at crossing angle $\beta=0.4$ with respect to the first pulse, and with $\Omega_{2} / \Omega_{1}=1 / 3$. The rotated full echo is formed at $\tau \approx T$ (namely, when $\sigma \tau=4.56$ ), and at angle $\theta=0.8(2 \beta)$. 


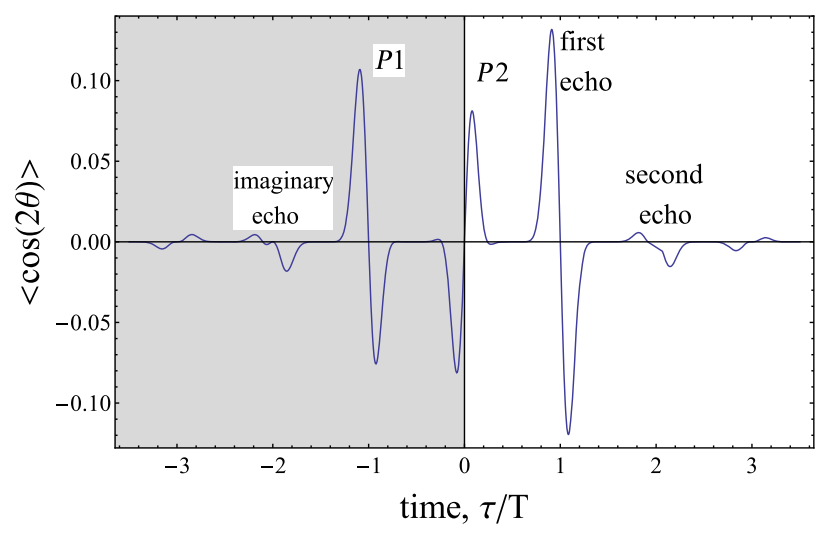

FIG. 4. Echoes for positive and negative time. Two pulses are applied at $\tau / T=-1$ and at $\tau=0$. Real echoes are seen at $\tau / T=1,2,3$ after the second pulse, and imaginary echoes are visible at $\tau / T=-2,-3$, i.e., at negative propagation time. The plotted quantity $\langle\cos (2 \theta)\rangle$ [Eq. (3), with $n=1$ ] is related to the standard alignment factor $\left\langle\cos ^{2} \theta\right\rangle$ by the standard trigonometric identity $\cos (2 \alpha)=2 \cos ^{2} \alpha-1$. $\theta=\beta+[(m \pi / T+\beta / T)] T(\bmod \pi)=2 \beta$. Thus, the echo will be not only delayed in time but also rotated by an additional angle $\beta$ with respect to the second pulse, or $2 \beta$ with respect to the first one (Fig. 3). Here, we report the first experimental observation of these rotated echoes (see Sec. III).

Imaginary echoes.-All the echoes discussed so far appear in the course of free evolution after the second pulse kicks the filamented phase-space pattern prepared by the first one. If, after the second kick, we were able to travel "back in time", the same qualitative arguments would predict the appearance of echoes at negative times $\tau$, clearly an impossibility in real physical systems. Fortunately, the phenomenon of quantum revivals [16-18] provides such an opportunity to probe "negative" times. The system dynamics just before the revival time corresponds to the classical evolution before $\tau=0$. Thus, we predict a series of echoes just before the revival of (a)
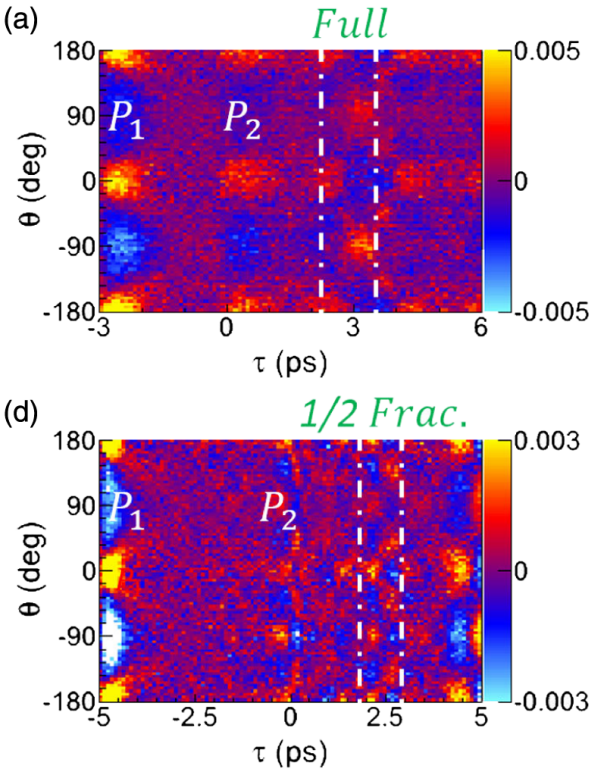

(g)

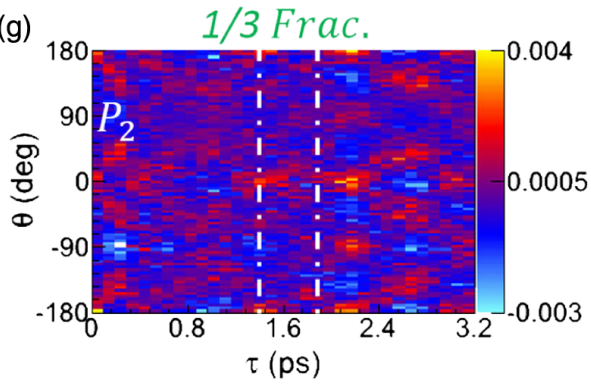

(b)

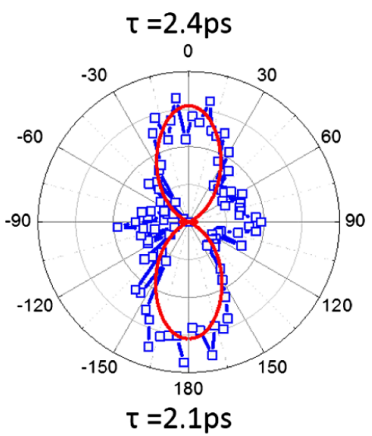

(e)

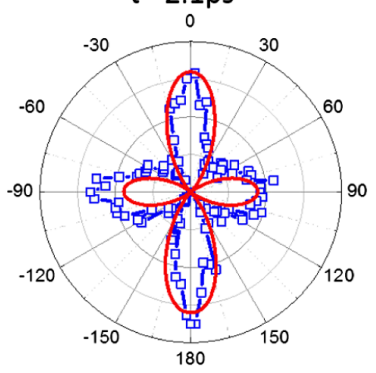

(h)

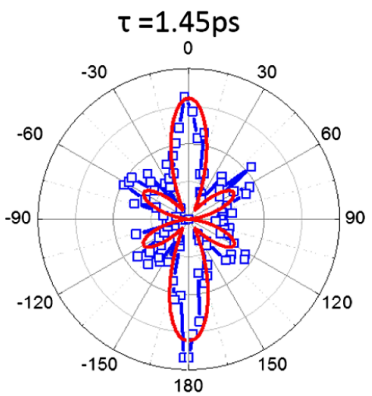

(c)

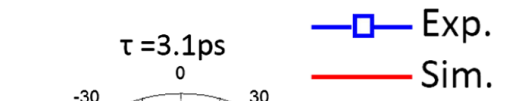

(f)

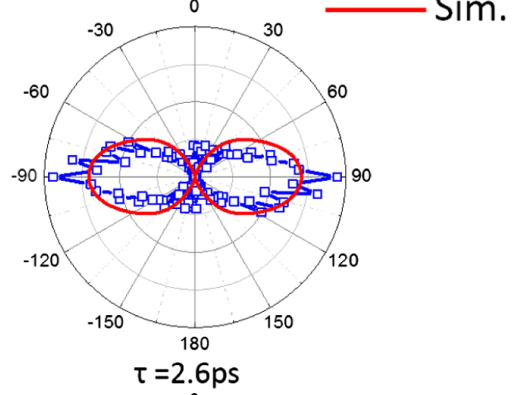

(i)
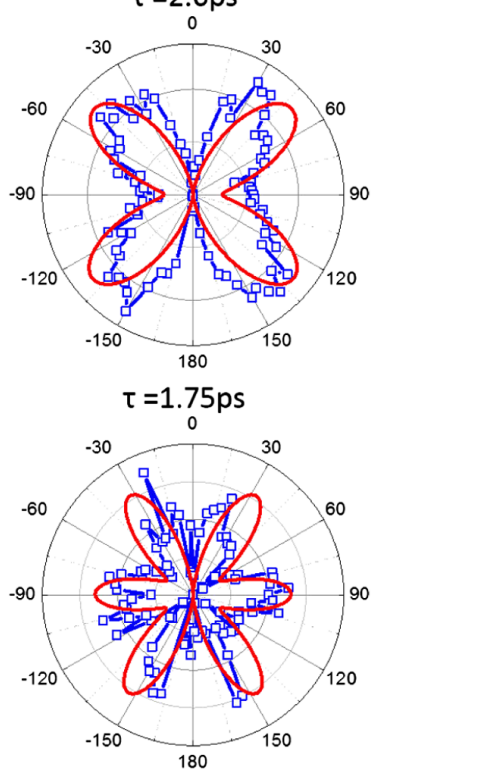

FIG. 5. Time-dependent angular distributions of full, $1 / 2$ and $1 / 3$ echoes, as indicated between the white dashed lines in (a) 2.2 ps$3.5 \mathrm{ps}$, (d) $1.9 \mathrm{ps}-3.0 \mathrm{ps}$, and (g) $1.4 \mathrm{ps}-1.9 \mathrm{ps}$, experimentally measured and theoretically simulated for $\mathrm{CO}_{2}$. (a) Carpet of the angular distribution of full echo for parallel input pulses, $P_{1}$ and $P_{2}$, with $T=3$ ps time delay between them. (b) and (c) Polar plots of the alignment and anti-alignment regions of the distribution for the full echo, showing vertical and horizontal directionality, respectively. (d)-(f) The same, for the $1 / 2$ fractional echo, with $T=5$ ps time delay. (g)-(i) The same for $1 / 3$ fractional echoes with $T=5$ ps time delay. In all cases, the solid red line is the quantum-mechanically simulated distribution. 
the first pump pulse. The shape of these echoes may be obtained from Eq. (3) by extending it to negative $\tau$. We term them "imaginary echoes" in line with Refs. [33,34], where related phenomena were first considered. Figure 4 presents echoes and imaginary echoes derived from Eq. (3) in a unified way.

\section{EXPERIMENTAL RESULTS}

Full and fractional echoes.-All the experimental results in this paper were obtained in our COLTRIMS setup, shown in Fig. 1, with details given in the Sec. 1 of the Appendix and in Ref. [27]. The raw data out of a COLTRIMS experiment are depicted as a "carpet" of probability distribution, where the horizontal axis is the probe time delay, $\tau$, measured from the second pulse; the vertical axis is the angle $\theta$ in the $Y-Z$ plane away from the $Z$ direction; and the density is given by the color code. To increase the visibility and eliminate the bias induced by possible imperfections in the circularity of the probe pulse, we normalize the total probability of the angular distribution to unity for each time delay and then subtract the averaged angular distribution between the two pump pulses. We start with the primary (full) alignment echo. In Fig. 5, the first pulse $\left(P_{1}\right)$ arrives at $\tau=-3$ ps and leads to the impulsive alignment of the molecular axis along the polarization direction of $P_{1}$, which vanishes with time because of the dispersion of molecular rotational velocities. A second pump $\left(P_{2}\right)$ polarized parallel to $P_{1}$ is applied at $\tau=0 \mathrm{ps}$ and leads to another immediate response. As discussed above, the following free rotational dynamics results in an alignment echo centered at twice the time delay after the first pulse, i.e., at $\tau=3$ ps. This echo is seen between the dashed lines in Fig. 5(a). The corresponding polar plot of the angular distribution around $\tau=2.4 \mathrm{ps}$ is shown in Fig. 5(b), demonstrating clear alignment along the same polarization direction as that of the exciting pulses. In previous optical studies of the alignment process after a double pulse excitation [19] (see also the related measurements of Ref. [35]), the ensemble-averaged alignment signal $\left\langle\cos ^{2} \theta\right\rangle$ was measured, while here (with the COLTRIMS methodology) we directly visualize the angular distribution of the molecules. As is clearly seen from the polar plot around $\tau=3.1 \mathrm{ps}$ [Fig. 5(c)], the echo not only shows alignment but also a rapid transition from the alignment to the anti-alignment. The red lines in Figs. 5(b) and 5(c) are the results of numerical simulations showing very good agreement with the measured signals. In Secs. 2 and 3 of the Appendix, we discuss both quantum-mechanical and classical simulations [see Figs. 9(a)-9(c) and Figs. 10(a)-10(c), respectively], and the two agree very well with one another and with the experimental results.

Besides the primary (full) echoes at $\tau=T$, and the higher-order full echoes at $\tau=2 T, 3 T \ldots$ (not shown), fractional echoes can now be clearly seenFigs. 5(d)-5(i) depict the situation for the $1 / 2$ and $1 / 3$ echoes at $\tau=T / 2$ and $T / 3$. To identify the temporal evolution of the fractional echoes, we increased the

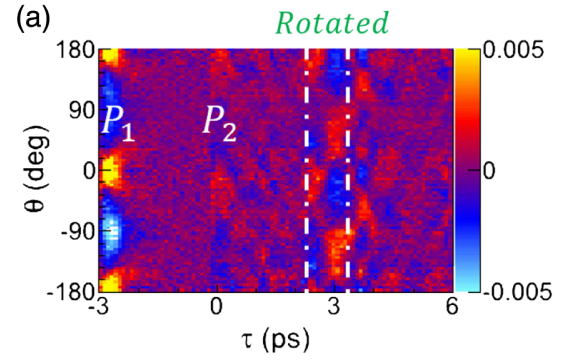

(b)

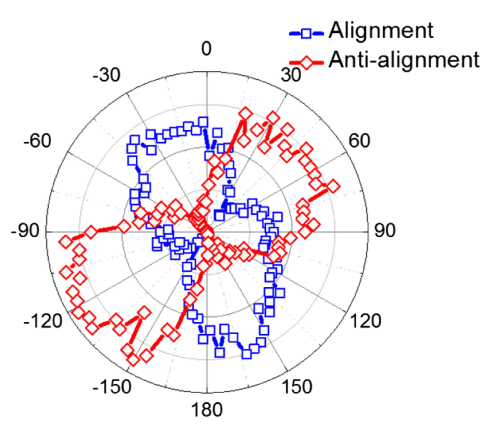

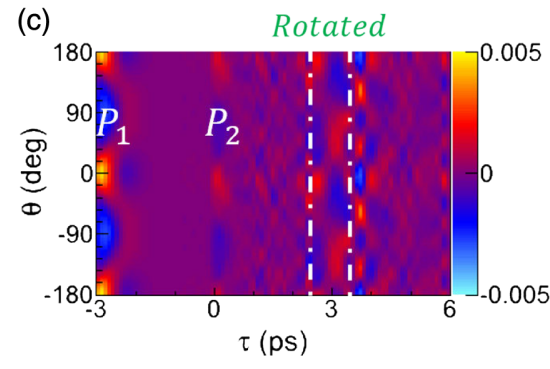

(d)

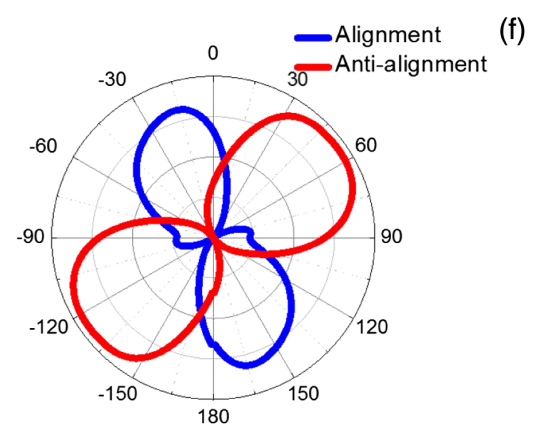

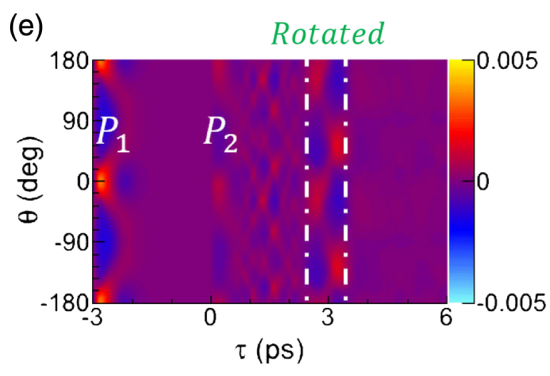

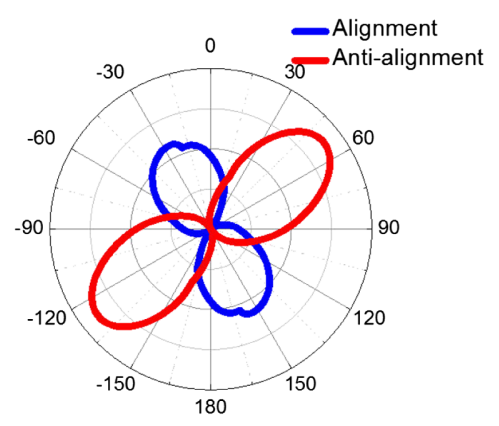

FIG. 6. Experimentally measured and theoretically simulated time-dependent angular distributions of the rotated echo in $\mathrm{CO}_{2} . P_{1}$ and $P_{2}$ arrive at -3 ps and $0 \mathrm{ps}$, respectively, with a crossing angle of $\beta=-20^{\circ}$. (a) Angular distribution of the rotated echo as a function of the probe time delay (see the region of 2.2-3.5 ps between the white dashed lines). (b) Polar plots of the rotated echo at 3 ps. Panels (c) and (d) show quantum simulations, and panels (e) and (f) show classical simulations. 
time delay between $P_{1}$ and $P_{2}$ to 5 ps. The measured time-dependent angular distribution of the $1 / 2$ fractional echo (emerging around $\tau=T / 2$ ) is shown in Figs. 5(d)-5(f). Whereas the full echo is cigar shaped, the fractional $1 / 2$ echo exhibits much more complex structures of a cross shape at $\tau=2.1 \mathrm{ps}$ followed by a butterfly distribution at $\tau=2.6 \mathrm{ps}$, as shown in Figs. 5(e) and 5(f). In Figs. 5(g)-5(i), we also visualize the $1 / 3$ echo. It emerges around $\tau=T / 3 \sim 1.66$ ps for $T=5 \mathrm{ps}$ and shows a more elaborate six-lobe molecular angular distribution. For both the fractional 1/2 and $1 / 3$ echoes, the experimental results shown in the polar plots are matched very well by the simulations (red in these figures), and here, too, the quantum-mechanical and classical simulations reproduce each other very

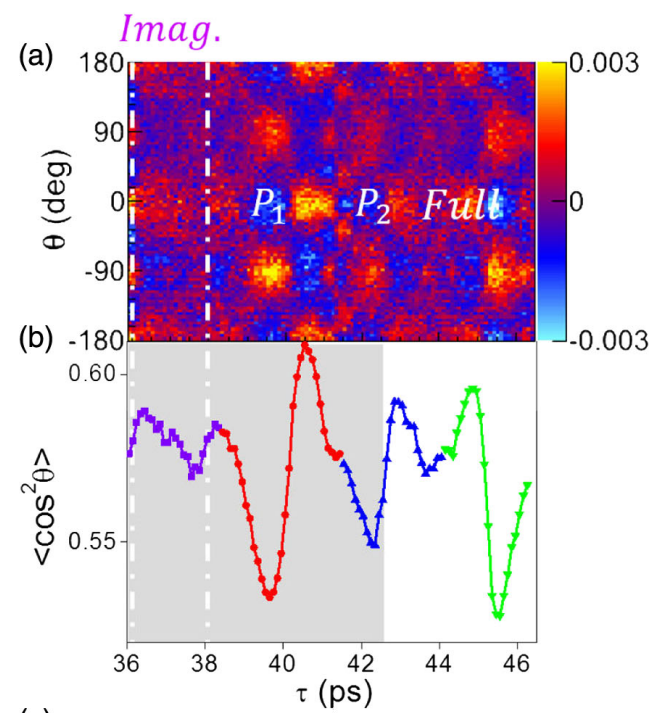

(c)

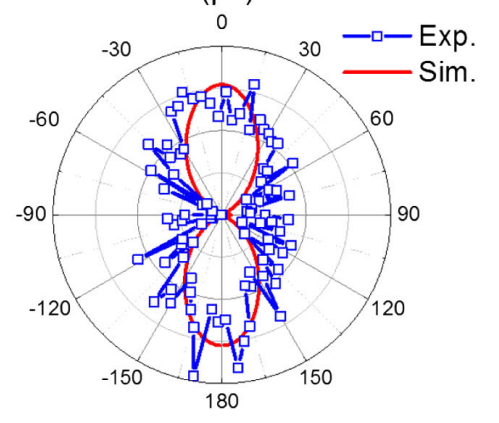

FIG. 7. Experimentally measured and theoretically simulated time-dependent angular distributions of the imaginary echo in $\mathrm{CO}_{2} . P_{1}$ and $P_{2}$ arrive at $-2.6 \mathrm{ps}$ and $0 \mathrm{ps}$, respectively. (a) A "carpet" describing the angular distribution of the imaginary echo as a function of the probe time delay (see the region of 36 ps -38 ps between the white dashed lines). (b) The alignment factor $\left\langle\cos ^{2} \theta\right\rangle$ as a function of the probe delay (red shows the revival of the response to the first pulse, blue that of the second pulse, green depicts the revival of the first full echo, and purple presents the imaginary echo). (c) Polar plots of the imaginary echo around $36.6 \mathrm{ps}$ (blue shows the experiment and red the simulation). well, as is discussed in detail in Secs. 2 and 3 of the Appendix [see Figs. 9(d)-9(i) and Figs. 10(d)-10(i), respectively].

Rotated and imaginary echoes.-A rotated echo that is caused by a time-delayed second pump pulse that is polarized at a crossing angle $\beta=-20^{\circ}$ with respect to the first one was observed. The measured angular distribution and its quantum and classical simulations are presented in Figs. 6(a)-6(f). The rotated full echo emerges around $\tau=3$ ps with the maximum of its (experimentally broadened) angular distribution first at $-40^{\circ}$ (alignment) followed by a rapid transfer to $-130^{\circ}$ (anti-alignment). As predicted above, the echo is rotated by $2 \beta$ with respect to the first pulse. This angular dependence of the rotated echo was further verified (not shown) by varying $\beta$ over a range

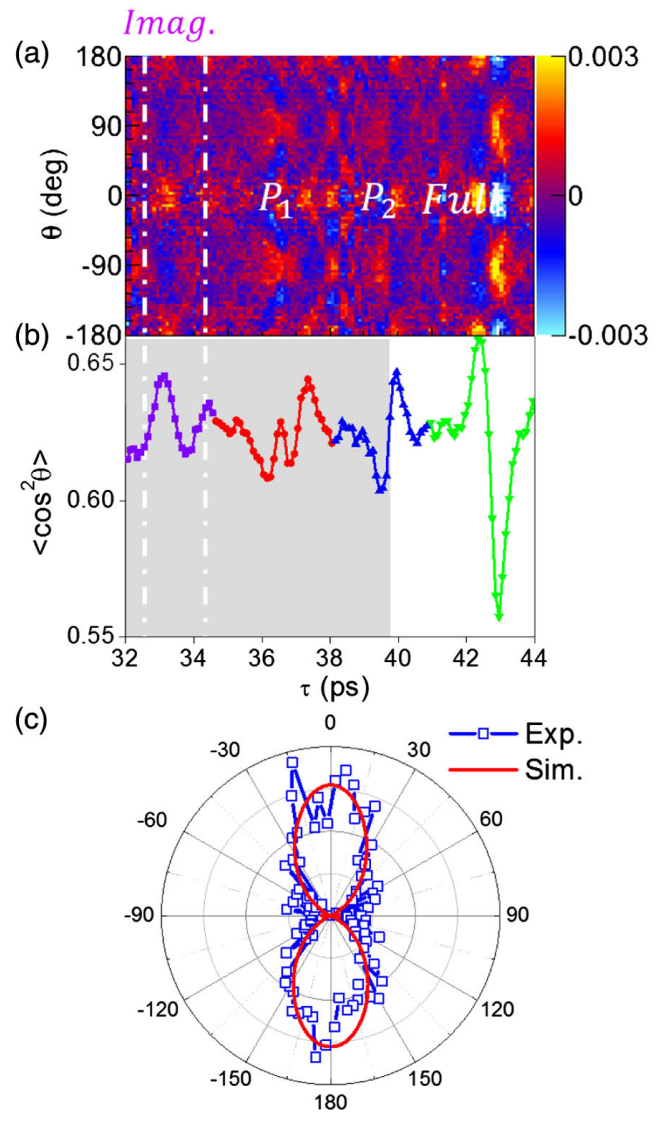

FIG. 8. Experimentally measured and theoretically simulated time-dependent angular distributions of the imaginary echo in $\mathrm{N}_{2} \mathrm{O}$. $P_{1}$ and $P_{2}$ arrive at -3 ps and 0 ps, respectively. (a) A "carpet" describing the angular distribution of the imaginary echo as a function of the probe time delay (see the region of 32.5-34.5 ps between the white dashed lines). (b) The alignment factor $\left\langle\cos ^{2} \theta\right\rangle$ as a function of the probe delay (red shows the revival of the response to the first pulse, blue that of the second pulse, green depicts the revival of the first full echo, and purple presents the imaginary echo). (c) Polar plots of the imaginary echo around 33 ps (blue is for experiment, red for simulation). 
of values and observing the corresponding angular dependence of the echo. These measurements provide the first spatiotemporal visualization of rotated echoes of impulsively aligned molecules. The entire simulated time evolution of the rotated echoes is described in the movie in Ref. [36].

The preceding results show that the time evolution of the twice-kicked ensemble gives rise to echoes that emerge after the application of the second laser pulse. Equation (3) predicts imaginary echoes for "negative" times before the first pulse, as depicted in Fig. 4. The time domain near (and earlier than) quantum full revival of the rotational excitation offers a unique opportunity to observe imaginary echoes at "negative" times.

Figure 7 shows (a) the time-dependent angular distribution and (b) the alignment factor for a double-pulse excited gas of $\mathrm{CO}_{2}$ molecules measured for long waiting times after the excitation. The red and blue peaks are the full quantum revival of the two pump pulses, the green signal is the quantum revival of the first full echo, and the purple peak around $36.6 \mathrm{ps}$ is the imaginary echo that precedes the revival of the first pulse by exactly the delay between the two exciting pulses.

$\mathrm{N}_{2} \mathrm{O}$ is a different molecule, with the rotational constant such that its revival time of $39.9 \mathrm{ps}$ is very similar to that of the $\mathrm{CO}_{2}$ molecule. Unlike $\mathrm{CO}_{2}$, because of different symmetry, this molecule does not demonstrate quarter revivals, and therefore, the time window before the full rotational revival provides more room for the observation of the imaginary echo. Figure 8 depicts a measurement near $32-44 \mathrm{ps}$ in $\mathrm{N}_{2} \mathrm{O}$. The replicas of the first and second pulses are clearly seen in the figure, both in the carpet [Fig. 8(a)] and in the alignment factor $\left\langle\cos ^{2} \theta\right\rangle(t)$ [Fig. 8(b)]. At earlier times, the imaginary echo is seen (marked by the two vertical lines), completely isolated from any neighboring signals. The polar plot of this imaginary echo is depicted in Fig. 8(c). (a)

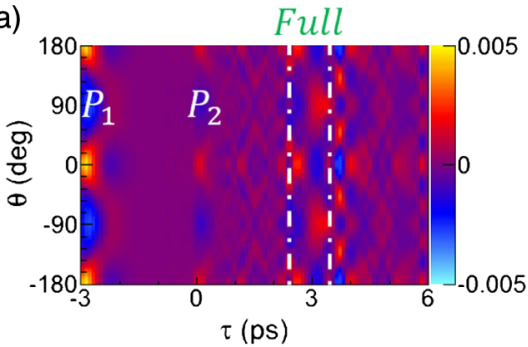

(d)

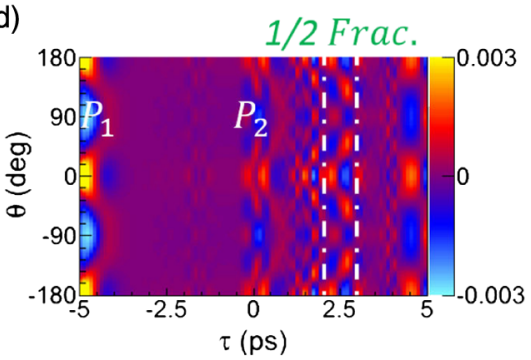

(g)

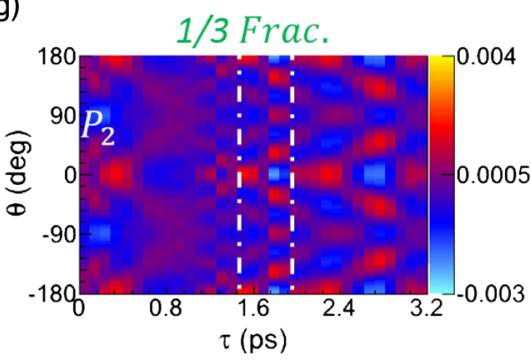

(b)

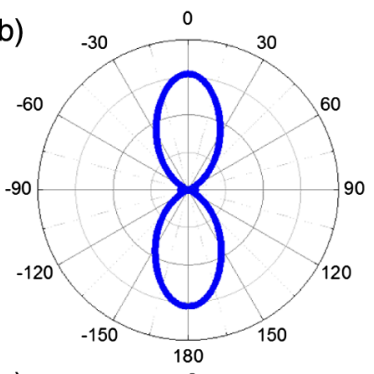

(e)

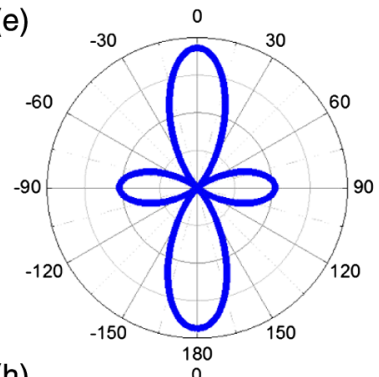

(h)

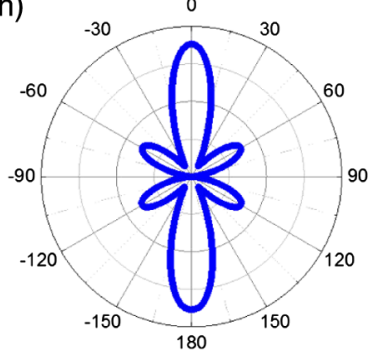

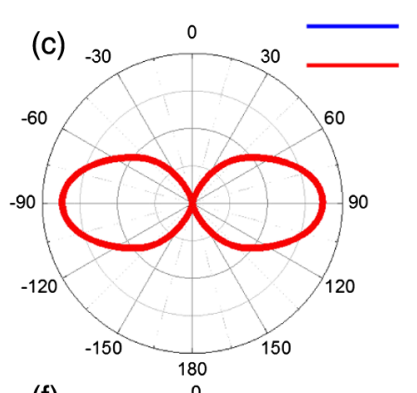

Alignment Anti-alignment
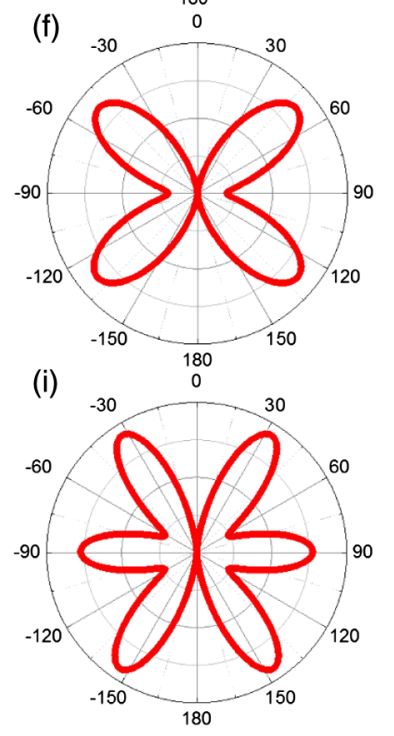

FIG. 9. Quantum simulation of time-dependent angular distributions of full, $1 / 2$, and $1 / 3$ echoes. (a) Angular distribution of full echo in the case of a parallel condition with a time delay of 3 ps between $P_{1}$ and $P_{2}$. (b,c) Alignment and anti-alignment of full echo in polar plots. Panels (d)-(f) and (g)-(i) show the same for $1 / 2$ and $1 / 3$ fractional echoes with a time delay of 5 ps between $P_{1}$ and $P_{2}$, respectively. 

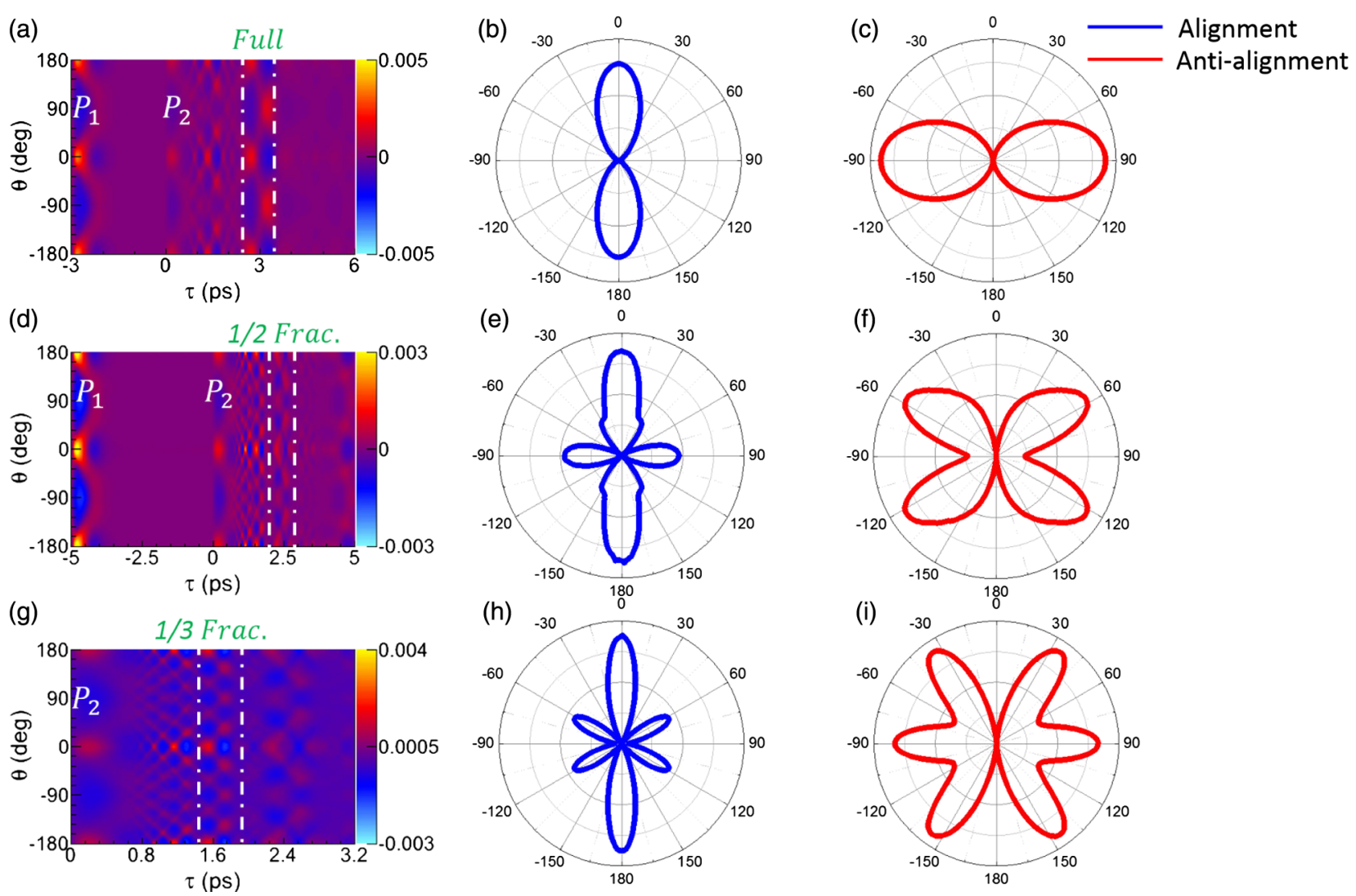

FIG. 10. Classical simulation of time-dependent angular distributions of full, 1/2, and 1/3 echoes. (a) Angular distribution of full echo in the case of a parallel condition with a time delay of 3 ps between $P_{1}$ and $P_{2}$. (b,c) Alignment and anti-alignment of full echo in polar plots. Panels (d)-(f) and (g)-(i) show the same for $1 / 2$ and $1 / 3$ fractional echoes with a time delay of 5 ps between $P_{1}$ and $P_{2}$, respectively.

\section{SUMMARY}

In this work, we analyze the echo phenomena in terms of phase-space filamentation and experimentally demonstrate the principles for a molecular rotational system. By using coincidence Coulomb-explosion imaging, we study the spatiotemporal dynamics of various alignment echoes. In addition to the full and fractional echoes, we report new types of echoes: rotated echoes and imaginary echoes. When a molecule is impulsively kicked by a pair of timedelayed and nonidentically polarized ultrashort laser pulses, the alignment echo appears at twice the time delay between the pumps, polarized along a direction that is at twice the angle between them. We predict these echoes, calculate them classically and quantum mechanically, and observe them experimentally. Furthermore, if time is formally allowed to flow backwards, imaginary echoes are predicted at negative times before the first pulse. To overcome the physical impossibility of working in negative time, we probe a time window just prior to the full quantum revival, which is fully equivalent to the window just prior to the origin. Imaginary echoes are indeed observed there.
The echo effects considered here are well described by classical analysis on short time scales and are actually of a classical origin. Since we study the echoes in a molecular system where quantum revivals are also present, it is important to distinguish between these two phenomena. While echoes and revivals both show recurrences of an original impulsive excitation, they are principally different. Quantum revivals [16-18], full or fractional, do not exist in classical systems, and they require energy quantization. They are observed after a single-pulse excitation, and their period is determined by the properties of the discrete energy spectrum. Echoes, on the other hand, appear only after two pulses and are common to many physical systems, including macroscopic ones, where classical mechanics is naturally applicable; their timing is determined by the delay between the two exciting pulses. In the molecular systems studied here, the interplay between the echoes and the quantum revivals shows up on a longer time scale (comparable with a considerable fraction of the rotational revival time), as demonstrated both in our experiments and in numerical simulations. 
The physics of laser-induced alignment or orientation of molecules [21-24] bears remarkable similarities to modern techniques for laser manipulations over electron beams in accelerator-based light sources [12-15]. In both cases, the goal is to compress the density of particles in certain areas (angular alignment or orientation and electron bunching along the beam) by folding the phase space with the help of laser pulses [12,15,37,38]. In particular, recent proposals to increase the electron-bunching factor by the use of several laser fields [15] are closely related to similar suggestions $[37,38]$ in the context of molecular orientation or alignment. Moreover, molecular alignment echoes are directly related to echo-enabled harmonics generation (EEHG) in freeelectron lasers [12-15]. The recently demonstrated efficient generation of high harmonics (up to the 75th) from tailored electron beams [39] is actually based on a mechanism similar to the fractional echoes of high order observed here. This realization of the analogy between seemingly different areas of physics may be beneficial for both fields.

\section{ACKNOWLEDGMENTS}

We appreciate useful discussions with Yaron Silberberg, Gennady Stupakov, and Dao Xiang. This work is supported by the National Natural Science Foundation of China (Grants No. 11374103, No. 11434005, and No. 11425416), Program of Introducing Talents of Discipline to Universities of China (B12024), the Conseil Régional de Bourgogne (PARI program), the CNRS, the French National Research Agency (ANR) through the CoConicS program (Contract No. ANR13-BS08-0013), and the Labex ACTION program (Contract No. ANR-11-LABX-0001-01). Support by the Israel Science Foundation (Grant No. 746/15), the ICORE program "Circle of Light," and the Minerva Foundation with funding from the Federal German Ministry for Education and Research is highly appreciated. I. A. acknowledges support as the Patricia Elman Bildner Professorial Chair. This research was made possible in part by the historic generosity of the Harold Perlman family.

\section{APPENDIX: METHODS AND SIMULATIONS}

\section{Methods}

The alignment echoes are generated in a molecular beam subject to a pair of femtosecond laser pulses, accompanied by an intense circularly polarized probe pulse that Coulomb-explodes the molecules to image their orientation at various time slices, as schematically shown in Fig. 1. The three pulses are derived from a linearly polarized femtosecond laser pulse $(25 \mathrm{fs}, 790 \mathrm{~nm}, 10 \mathrm{kHz}$, Ti:sapphire Femtolasers) and are denoted as $P_{1}, P_{2}$, and probe pulse, respectively. Two individual half-wave plates are placed in the optical path of the pulses to polarize $P_{1}$ along the $\mathrm{Z}$ axis and to adjust the polarization of $P_{2}$ for the different experiments. The three pulses are focused onto a supersonic molecular beam in an ultrahigh vacuum chamber of the
COLTRIMS apparatus by a concave silver mirror ( $\mathrm{f}=7.5 \mathrm{~cm}$ ). The temporal duration of each pulse in the interaction region is estimated to be about $60 \mathrm{fs}$ by tracing the time-delay-dependent single-ionization yield as a crosscorrelation of every two pulses. To achieve strong echo signals, the intensity ratio of $I_{2} / I_{1}$ in the case of parallel polarizations of $P_{2}$ and $P_{1}$ is experimentally optimized to around 0.3. The intensities of $P_{1}$ and $P_{2}$ in the reaction area are measured to be $I_{1} \sim 5.0 \times 10^{13} \mathrm{~W} / \mathrm{cm}^{2}$ and $I_{2} \sim 1.4 \times 10^{13} \mathrm{~W} / \mathrm{cm}^{2}$, and the intensity of the probe pulse is about $4 \times 10^{14} \mathrm{~W} / \mathrm{cm}^{2}$. The produced fragment ions are accelerated and guided by a weak homogeneous static electric field (about $20 \mathrm{~V} / \mathrm{cm}$ ) and then detected by a time- and position-sensitive microchannel plate detector. In general, the three-dimensional momenta of the ions are retrieved from the measured times of flights and positions of impacts. In our case of linear molecules, the direction of the molecular axis is retrieved from the double-ionization-induced Coulombexplosion channel of $\left[\mathrm{CO}_{2}+n \hbar \omega \rightarrow \mathrm{CO}^{+}+\mathrm{O}^{+}+2 e\right]$ and $\left[\mathrm{N}_{2} \mathrm{O}+n \hbar \omega \rightarrow \mathrm{NO}^{+}+\mathrm{N}^{+}+2 e\right]$ for $\mathrm{CO}_{2}$ and $\mathrm{N}_{2} \mathrm{O}$, respectively. The angle $\theta$ away from the $Z$ axis (in the $Y-Z$ plane) is tracked as a function of probe-pulse delay to reveal the evolution of the molecular orientation. The probe pulse is circularly polarized in the $Y-Z$ plane, and we restrict our data analysis in this plane by selecting molecules confined to $\left[-20^{\circ}, 20^{\circ}\right]$ with respect to the $Y-Z$ plane (see Fig. 1).

\section{Quantum-mechanical simulation}

We model the alignment echo by numerically solving the time-dependent Schroedinger equation $i \hbar \partial|\psi\rangle / \partial t=$ $H_{\text {eff }}|\psi\rangle$ for the rotational state $|\psi\rangle=\sum_{\mathrm{J}, \mathrm{M}} C_{\mathrm{JM}}|J M\rangle$, where $H_{\text {eff }}=B_{0} J(J+1)-0.5 \Delta \alpha \sin ^{2} \theta\left(\varepsilon_{z}^{2} \cos ^{2} \phi+\varepsilon_{y}^{2} \sin ^{2} \phi+\right.$ $\left.2 \varepsilon_{z} \varepsilon_{y} \cos \phi \sin \phi\right)$ is the effective Hamiltonian, $B_{0}$ is the molecular rotational constant, $\Delta \alpha$ is the difference between the polarizability components parallel and perpendicular to the molecular axis, $\theta$ and $\phi$ are the polar and azimuth angles of the molecular axis with respect to the $X$ and $Z$ axes, and $\varepsilon_{y}$ and $\varepsilon_{z}$ are the envelopes of the laser field vector along the $Y$ and $Z$ axes, respectively. We first calculate the term $P_{J_{0} M_{0}}(\theta, \phi, t)=\left|\sum_{\mathrm{J}, \mathrm{M}} C_{\mathrm{JM}}(t) Y_{\mathrm{JM}}(\theta, \phi)\right|^{2}$ for each initial molecular rotational state $\left|\psi_{(t=0)}\right\rangle_{J_{0} M_{0}}=\left|J_{0}, M_{0}\right\rangle$, where $Y_{\mathrm{JM}}(\theta, \phi)$ are the spherical harmonic functions. We then assemble them by considering the temperature-dependent Boltzmann distribution of the initial rotational states and the proper spin statistics, and obtain the time-dependent probability density distribution of the rotational wave packet $P(\theta, \phi, t)$. For example, the molecular parameters of $\mathrm{CO}_{2}$ are $B_{0}=0.39 \mathrm{~cm}^{-1}, \Delta \alpha=2.0 \AA^{3}$. The rotational temperature of the molecular beam is very close to the translation temperature, which can be experimentally estimated from $T_{\text {trans }}=\Delta p^{2} /\left[4 \ln (4) k_{B} m\right]$, where $k_{B}$ is the Boltzmann constant, and $\Delta p$ and $m$ are the full width at half maximum (FWHM) of the momentum distribution (in the jet direction, i.e., $Y$ axis) and mass of the singly ionized 
$\mathrm{CO}_{2}^{+}$, respectively. In our experiment, we measure a momentum width in the jet direction of $\Delta p \sim 10.4$ a.u. of $\mathrm{CO}_{2}^{+}$ions created by $P_{1}$ polarized along the $Z$ axis (orthogonally to the gas jet). The initial rotational temperature of the $\mathrm{CO}_{2}$ molecule is estimated to be $75 \mathrm{~K}$, which shows good agreement with the experimental observations by matching the calculated arrival time of the first alignment maximum as well as those of the fractional revivals after the excitation of $P_{1}$. The intensities of $P_{1}$ and $P_{2}$ are chosen to match the experiments well.

\section{Classical simulation}

We first initialize the rotational parameters of each molecule $m$ of an ensemble of $N_{m}$ molecules, i.e., the molecule axis orientation vector $\vec{u}_{m}(t=0)$ and angular momentum $\vec{\omega}_{m}(t=0)$. This is done using Maxwell Boltzmann statistics at the chosen temperature for $\left\|\vec{\omega}_{m}(t=0)\right\|$, and random orientations for $\vec{u}_{m}(t=0)$ and $\vec{\omega}_{m}(t=0)$ [with $\vec{\omega}_{m}(t=0) \perp \vec{u}_{m}(t=0)$ ]. The molecules are then free to rotate, a situation where $\vec{\omega}_{m}(t+d t)=$ $\vec{\omega}_{m}(t)$ and $\vec{u}_{m}(t+d t)$ is easily obtained from $\vec{u}_{m}(t)$ and $\vec{\omega}_{m}(t=0)$, except during the laser pulses. In this latter case, we first compute, for each molecule $m$, the polarizability tensor $\overrightarrow{\vec{\alpha}}_{m}^{L}(t)$ in the laboratory fixed frame from that $\overrightarrow{\vec{\alpha}}_{M}$, in the molecular frame: $\overrightarrow{\vec{\alpha}}_{m}^{L}(t)=R_{m}(t) \cdot \overrightarrow{\vec{\alpha}}_{M}$. $R_{m}^{-1}(t)$, where $R_{m}(t)$ is the rotation matrix connecting the two frames [obtained from the orientation of the molecule axis $\left.\vec{u}_{m}(t)\right]$. The dipole $\vec{\mu}_{m}(t)$ induced in the molecule by the laser field $\vec{E}(t)$ is then $\vec{\mu}_{m}(t)=$ $\overrightarrow{\vec{\alpha}}_{m}^{L}(t) \cdot \vec{E}(t)$, from which the torque $\vec{\tau}_{m}(t)$ subsequently applied to the molecule is computed from $\vec{\tau}_{m}(t)=$ $\mu_{m}(t) \wedge \vec{E}(t)$. This enables the calculation of $d \vec{\omega}_{m} / d t(t)=$ $\vec{\tau}_{m}(t) / I$, where $I$ is the molecule moment of inertia, from which $\vec{\omega}_{m}(t+d t)=\vec{\omega}(t)+d t \times d \vec{\omega}_{m} / d t(t)$ is obtained, enabling us to deduce $\vec{u}_{m}(t+d t)$ from $\vec{u}_{m}(t)$, and so on. Note that, since the period of the laser field oscillation is much shorter than the laser pulse duration and the molecule rotational period, the $|\vec{E}(t)|^{2}$ appearing in the expression of the torque can be replaced by $\varepsilon^{2}(t) / 2$, where $\varepsilon(t)$ is the field envelope. Furthermore, in order to correctly sample the associated multidimensional phase space and obtain sufficiently converged results, several tens of millions of molecules must be used. A sufficiently small time step $d t$, with respect to the laser pulse(s) duration and molecular rotation period, should obviously be used.

[1] E. L. Hahn, Spin Echoes, Phys. Rev. 80, 580 (1950).

[2] E. L. Hahn, Free Nuclear Induction, Phys. Today 6 No. 11, 4 (1953).

[3] N. A. Kurnit, I. D. Abella, and S. R. Hartmann, Observation of a Photon Echo, Phys. Rev. Lett. 13, 567 (1964).
[4] R. M. Hill and D. E. Kaplan, Cyclotron Resonance Echo, Phys. Rev. Lett. 14, 1062 (1965).

[5] R. W. Gould, T. M. O’Neil, and J. H. Malmberg, Plasma Wave Echo, Phys. Rev. Lett. 19, 219 (1967).

[6] A. Bulatov, A. Kuklov, B. E. Vugmeister, and H. Rabitz, Echo in Optical Lattices: Stimulated Revival of Breathing Oscillations, Phys. Rev. A 57, 3788 (1998).

[7] M. Herrera, T. M. Antonsen, E. Ott, and S. Fishman, Echoes and Revival Echoes in Systems of Anharmonically Confined Atoms, Phys. Rev. A 86, 023613 (2012).

[8] T. Meunier, S. Gleyzes, P. Maioli, A. Auffeves, G. Nogues, M. Brune, J. M. Raimond, and S. Haroche, Rabi Oscillations Revival Induced by Time Reversal: A Test of Mesoscopic Quantum Coherence, Phys. Rev. Lett. 94, 010401 (2005).

[9] G. V. Stupakov, Echo Effect In Hadron Colliders, SSC Report SSCL-579, 1992, http://www.osti.gov/scitech/ servlets/purl/7237216/.

[10] L. K. Spentzouris, J.-F. Ostiguy, and P. L. Colestock, Direct Measurement of Diffusion Rates in High Energy Synchrotrons Using Longitudinal Beam Echoes, Phys. Rev. Lett. 76, 620 (1996).

[11] R. W. Brown, Y.-C. N. Cheng, E. M. Haacke, M. R. Thompson, and R. Venkatesan, Magnetic Resonance Imaging: Physical Principles and Sequence Design (Wiley-Blackwell, New York, 2014).

[12] G. Stupakov, Using the Beam-Echo Effect for Generation of Short-Wavelength Radiation, Phys. Rev. Lett. 102, 074801 (2009).

[13] D. Xiang, E. Colby, M. Dunning, S. Gilevich, C. Hast, K. Jobe, D. McCormick, J. Nelson, T. O. Raubenheimer, K. Soong, G. Stupakov, Z. Szalata, D. Walz, S. Weathersby, and M. Woodley, Demonstration of the Echo-Enabled Harmonic Generation Technique for Short-Wavelength Seeded Free Electron Lasers, Phys. Rev. Lett. 105, 114801 (2010).

[14] Z. T. Zhao, D. Wang, J. H. Chen, Z. H. Chen, H. X. Deng, J. G. Ding, C. Feng, Q. Gu, M. M. Huang, T. H. Lan et al., First Lasing of an Echo-Enabled Harmonic Generation Free-Electron Laser, Nat. Photonics 6, 360 (2012).

[15] E. Hemsing, G. Stupakov, D. Xiang, and A. Zholents, Beam by Design: Laser Manipulation of Electrons in Modern Accelerators, Rev. Mod. Phys. 86, 897 (2014).

[16] J. Parker and C. R. Stroud, Jr., Coherence and Decay of Rydberg Wave Packets, Phys. Rev. Lett. 56, 716 (1986).

[17] I. Sh. Averbukh and N. F. Perelman, Fractional Revivals: Universality in the Long-Term Evolution of Quantum Wave Packets Beyond the Correspondence Principle Dynamics, Phys. Lett. 139A, 449 (1989).

[18] R. W. Robinett, Quantum Wave Packet Revivals, Phys. Rep. 392, 1 (2004).

[19] G. Karras, E. Hertz, F. Billard, B. Lavorel, J.-M. Hartmann, O. Faucher, E. Gershnabel, Y. Prior, and I. Sh. Averbukh, Orientation and Alignment Echoes, Phys. Rev. Lett. 114, 153601 (2015).

[20] G. Karras, E. Hertz, F. Billard, B. Lavorel, G. Siour, J.-M. Hartmann, O. Faucher, E. Gershnabel, Y. Prior, and I. Sh. Averbukh, Experimental Observation of Fractional Echoes, Phys. Rev. A 94, 033404 (2016).

[21] H. Stapelfeldt and T. Seideman, Aligning Molecules with Strong Laser Pulses, Rev. Mod. Phys. 75, 543 (2003). 
[22] Y. Ohshima and H. Hasegawa, Coherent Rotational Excitation by Intense Nonresonant Laser Fields, Int. Rev. Phys. Chem. 29, 619 (2010).

[23] S. Fleischer, Y. Khodorkovsky, E. Gershnabel, Y. Prior, and I. Sh. Averbukh, Molecular Alignment Induced by Ultrashort Laser Pulses and Its Impact on Molecular Motion, Isr. J. Chem. 52, 414 (2012).

[24] M. Lemeshko, R. V. Krems, J. M. Doyle, and S. Kais, Manipulation of Molecules with Electromagnetic Fields, Mol. Phys. 111, 1648 (2013).

[25] A. J. Lichtenberg, Phase-Space Dynamics of Particles (Wiley, New York, 1969).

[26] R. Dörner, V. Mergel, O. Jagutzki, L. Spielberger, J. Ullrich, R. Moshammer, and H. Schmidt-Böcking, Cold Target Recoil Ion Momentum Spectroscopy: A "Momentum Microscope" to View Atomic Collision Dynamics, Phys. Rep. 330, 95 (2000).

[27] K. Lin, Q. Y. Song, X. C. Gong, Q. Y. Ji, H. F. Pan, J.X. Ding, H. P. Zeng, and J. Wu, Visualizing Molecular Unidirectional rotation, Phys. Rev. A 92, 013410 (2015).

[28] K. Mizuse, K. Kitano, H. Hasegawa, and Y. Ohshima, Quantum Unidirectional Rotation Directly Imaged with Molecules, Sci. Adv. 1, e1400185 (2015).

[29] R. W. Boyd, Nonlinear Optics (Academic Press, New York, 1992).

[30] B. Friedrich and D. Herschbach, Alignment and Trapping of Molecules in Intense Laser Fields, Phys. Rev. Lett. 74, 4623 (1995); Polarization of Molecules Induced by Intense Nonresonant Laser Fields, J. Phys. Chem. 99, 15686 (1995).

[31] A. J. Lichtenberg and M. A. Lieberman, in Regular and Chaotic Dynamics, edited by J. E. Marsden and L. Sirovich,
Applied Mathematical Sciences (Springer-Verlag, New York, 1992).

[32] D. Lynden-Bell, Statistical Mechanics of Violent Relaxation in Stellar Systems, Mon. Not. R. Astron. Soc. 136, 101 (1967).

[33] B. Ya. Dubetskii and V. P. Chebotaev, Echoes in Classical and Quantum Ensembles with Determinate Frequencies, Pis'ma Zh. Eksp. Teor. Fiz. 41, 267 (1985) [JETP Lett. 41, 328 (1985)].

[34] B. Ya. Dubetskii and V. P. Chebotaev, Imaginary Echo in a Gas in a Doppler Expanded Transition, Izv. Akad. Nauk SSSR, Ser. Fiz. 50, 1530 (1986) [Bull. Acad. Sci. USSR, Phys. Ser. (English Transl.) 50, 70 (1986)].

[35] H. Y. Jiang, C. Y. Wu, H. Zhang, H. B. Jiang, H. Yang, and Q. H. Gong, Alignment Structures of Rotational Wavepacket Created by Two Strong Femtosecond Laser Pulses, Opt. Express 18, 8990 (2010).

[36] See Supplemental Material at http://link.aps.org/ supplemental/10.1103/PhysRevX.6.041056 for a movie illustrating the formation of a rotated echo.

[37] I. Sh. Averbukh and R. Arvieu, Angular Focusing, Squeezing, and Rainbow Formation in a Strongly Driven Quantum Rotor, Phys. Rev. Lett. 87, 163601 (2001).

[38] M. Leibscher, I. Sh. Averbukh, P. Rozmej, and R. Arvieu, Semiclassical Catastrophes and Cumulative Angular Squeezing of a Kicked Quantum Rotor, Phys. Rev. A 69, 032102 (2004).

[39] E. Hemsing, M. Dunning, B. Garcia, C. Hast, T. Raubenheimer, G. Stupakov, and D. Xiang, Echo-Enabled Harmonics up to the 75th Order from Precisely Tailored Electron Beams, Nat. Photonics 10, 512 (2016). 